13.01.-15.01.2011

Berlin

Deutschland

\subsection{1-22.01.2011}

Berlin

Deutschland

-...................

21.01.-23.01.2011

Frankfurt/Main

Deutschland

...............

28.01.-29.01.2011

Berlin

Deutschland

.................

19.02.2011

Ludwigshafen

Deutschland

•...............

24.02.-26.02.2011

St. Gallen

Schweiz

................

24.02.-25.02.2011

Frankfurt/Main

Deutschland

•..............

26.02.2011

Frankfurt/Main

Deutschland

000000000000.000

25.02-27.02.2011

München

Deutschland

•.............

16.03.-19.03.2011

St. Gallen

Schweiz

[... . . . . . . . .

25.03.-26.03.2011

Berlin

Deutschland

•・・・・・・・・

01.04.-02.04.2011

Hamburg

Deutschland

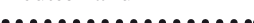

07.04.-09.04.2011

Tübingen

Deutschland

\subsection{4-30.04.2011}

Warth b. Frauenfeld

Schweiz

.................

03.06.-07.06.2011

Chicago

USA

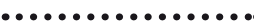

16.06.-18.06.2011

Berlin

Deutschland

•................

25.08.-26.08.2011

St. Gallen

Schweiz

................

30.09.-04.10.2011

Basel

Schweiz 5th International Symposium on the Biology and Immunology

of Cutaneous Lymphomas

5. Jahrestagung der Gesellschaft für Gastroenterologie und Hepatologie in Berlin und Brandenburg e.V. (GGHBB)

KAM-Seminar 2011 - Komplementäre und Alternative Therapien -

im Rahmen des deutschsprachigen ESO-Programms

6. Onkologie-Update-Seminar "Onko Update 2011»

V. "LUKS" Ludwigshafener und Kölner Stentworkshop

21. Ärzte-Fortbildungskurs in Klinischer Onkologie

Jahrestreffen der «German Breast Group» 2011

AGO «State of the Art Meeting" 2011

Zertifizierungskurs Medikamentöse Tumortherapie in der

Dermato-Onkologie

12th International St. Gallen Breast Cancer Conference

2. ASORS-Jahreskongress Supportive Therapie und Rehabilitation

bei Krebs - State of the Art 2011

8. Hamburger Update Gynäkologie und Geburtshilfe

52. Jahrestagung der Südwestdeutschen Gesellschaft für Urologie e.V.

15. Internationales Seminar: Pallativbetreuung von Tumorkranken

\section{7th ASCO Annual Meeting}

\section{State of the Art Symposioum AGO}

14. Internationales Seminar: Onkologische Pflege - Fortgeschrittene Praxis

\section{DGHO 2011}

\section{Auskunft:}

CTW Congress Organisation Thomas Wiese GmbH michael.jander@ctw-congress.de

www.ctw-congress.de

\section{Auskunft:}

www.gghbb.de

www.cocs.de

Auskunft:

www.uct-frankfurt.de

\section{Auskunft:}

www.onko-update.com

\section{Auskunft: $\mathrm{COCS} \mathrm{GmbH}$}

Eva.gautsch@cocs.de

www.cocs.de

Auskunft: Kongress-Sekretariat/ deso

deso@oncoconferences.ch

www.kssg.ch

Auskunft: GBG Forschungs $\mathrm{GmbH}$

Event@GermanBreastGroup.de

www.germanbreastgroup.de

Auskunft: GBG Forschungs $\mathrm{GmbH}$

Event@GermanBreastGroup.de

www.ago-online.org

Auskunft: MedConcept $\mathrm{GmbH}$

info@medconcept.org

www.medconcept.or

Auskunft: www.oncoconferences.ch www.zetup.ch

Auskunft: www.kongresseonline.de/ASORS_2011

Auskunft: www.hhgyn.de

Auskunft: INTERPLAN Congress, Meeting \& Event

Management AG

f.simon@interplan.de

www.interplan.de

Auskunft: Kongress-Sekretariat/ deso

deso@oncoconferences.ch

www.kssg.ch

Auskunft: www.chicago $2011 . a s c 0.0 r g$

Auskunft: If-kongress management gmbh info@if-kongrinfo@if-kongress.de

www.if-kongress.de

.................

Auskunft: Kongress-Sekretariat/ deso

deso@oncoconferences.ch

www.kssg.ch

Auskunft: DGHO Service GmbH www.haematologie-onkologie-2011.ch

\section{KARGER}

Fax +49 7614520714

Information@Karger.de

www.karger.com

\section{(c) 2010 S. Karger GmbH, Freiburg}

